\title{
ON SUPERCONDUCTING TRANSITION TEMPERATURE IN AMORPHOUS ALLOYS OF SIMPLE METALS
}

\author{
Yu.P. Krasny ${ }^{a}$, N.P. KovalenKo ${ }^{b}$, J. KrawczyK ${ }^{a}$ \\ AND E.P. GURNITSKAJA ${ }^{c}$ \\ ${ }^{a}$ Opole University, Oleska 48, 45-052 Opole, Poland \\ ${ }^{b}$ I.I. Mechnikov State University, Dvorianskya 2, 270100 Odessa, Ukraine \\ ${ }^{c}$ M.V. Lomonosov Technology Institute, Odessa, Ukraine \\ (Received November 13, 1998; revised version March 26, 1999; \\ in final form May 31, 1999) \\ The Eliashberg equations are generalised to describe amorphous binary \\ alloys. Equations for the electron-phonon coupling constant $\lambda$ are obtained. \\ An additional term caused by topological disorder appears in the expres- \\ sion for $\lambda$-constant. The features of amorphous state and their influence on \\ $\lambda$-constant and superconducting transition temperature are emphasised and \\ analysed. Theoretical results are illustrated by numerical calculations per- \\ formed for some binary amorphous alloys of simple metals.
}

PACS numbers: 74.62.-c, 74.70.Ad

\section{Introduction}

Numerous experiments indicate the influence of disorder on the superconducting transition temperature $T_{\mathrm{c}}$ in crystalline and amorphous metals and their compounds [1-11]. The presence of nonmagnetic impurities in simple metals or transition from the crystalline state to the amorphous one causes usually an enhancement of $T_{\mathrm{c}}$ [1-11]. It is a rather small effect, typically of the order of $10-20 \%$. However, several examples of a more drastic increase in $T_{c}$ with inserting disorder can be presented $[12,13]$.

One can distinguish two trends in the first theoretical attempts to explain disorder-induced changes of $T_{c}$. Some authors concentrated their efforts on investigations of the electron-phonon interaction in dirty superconductors while others dealt with the Coulomb pseudopotential only. In their pioneering work [14] Keck and Schmid have shown that nonmagnetic impurities change the electron-phonon 
interaction in a metal. They derived the Eliashberg equations for impure simple metals which had the same formal structure as in the clean metal case. An additional coupling of low-frequency transverse phonons to the electrons appears owing to the impurities. Therefore, the effective phonon density of state is considerably enlarged in the low-frequency region, which can lead to an increased transition temperature $T_{\mathrm{c}}[14]$.

Anderson et al. [15] considered the clisorder effect on the Coulomb pseudopotential $\mu^{*}$. Assuming that the phonon parts of the self-energies do not change, they have found a small correction to $\mu^{*}$ because of the crystal defect [15]. A strong enhancement of $\mu^{*}$ takes place just in the regime of the Anderson transition [15].

The results of Refs. $[14,15]$ as well as $[16,17]$ were generalized by Belitz [18]. One of the main conclusions [18] is the following: an increase in $T_{\mathrm{c}}$ with disorder occurs for a weak coupling and a decrease in $T_{\mathrm{c}}$ is observed for a strong coupling. This is in accord with the experimental facts.

The problem of mutual interplay of the Anderson localization and superconductivity in strongly disordered systems is analyzed in a review [19]. Superconductivity can occur close to the metal-insulator transition in some systems. A number of experiments demonstrating superconductivity close to the Anderson transition both in "traditional" and high- $T_{\mathrm{c}}$ superconductors is discussed in Ref. [19].

The problem of the superconducting transition in amorphous metals has some specific features [20-22]. A model of frozen liquid was exploited in Refs. [21, 22] in solving this problem. In comparison with the case of a crystalline ideal metal an additional term $\lambda_{1}$, taking into account interaction of electrons with the transverse phonons, appears in the expression for the electron-phonon coupling constant. $\lambda[20-22]$. Thus, the situation in a clean amorphous metal is very much similar to the case of a crystalline impure metal [14]. One should emphasize that the expression for the $\lambda_{1}$ term given in Refs. [20-22] can be derived from the appropriate equations obtained in Ref. [14], if the Ziman [23] formula for the time relaxation was used.

The authors of Refs. [20-22] draw also attention to another mechanism of the increase in the electron-phonon coupling constant which is specific for the amorphous metals only. The presence of the roton-like minimum on the dispersion phonon curves in the short-wave region may cause an enhancement of $\lambda$ in amorphous metals [21].

The extension of the previous results [20-22] to the case of amorphous binary alloys is the aim of the present paper which is organized as follows. Equations for the electron-phonon coupling constant $\lambda$ are derived in Sec. 2. Numerical illustrations of the theory and their discussion are given in Sec. 3. Calculations performed for some amorphous binary alloys of simple metals indicate that the superconducting transition temperature $T_{c}$, as a function of alloy concentration can have a maximum $T_{\mathrm{c}}^{\mathrm{m}}$. In some cases $T_{\mathrm{c}}^{\mathrm{m}}$ may exceed the $T_{\mathrm{c}}$ values of pure amorphous metals forming this alloy. 


\section{Basic equations}

As follows from Refs. $[14,18,24]$, the presence of disorder in a system does not change the formal structure of the Eliashberg equations except modification of the Eliashberg function $\alpha^{2}(\omega) g(\omega)$ form. The $\alpha^{2}(\omega) g(\omega)$ function is related to the electron-phonon coupling constant $\lambda$ by the following equation $[14,18,20-22]$ :

$$
\lambda=2 \int_{0}^{\infty} \frac{\mathrm{d} \omega}{\omega} \alpha^{2}(\omega) g(\omega)
$$

That is why one can use the well-known McMillan formula determining the temperature of the superconducting transition $[25,26]$

$$
T_{\mathrm{c}}=\frac{\Theta_{\mathrm{D}}}{1.45} \exp \left[-\frac{1.04(1+\lambda)}{\lambda-\mu^{*}(1+0.62 \lambda)}\right]
$$

where $\Theta_{\mathrm{D}}$ is the Debye function, $\mu^{*}$ - the effective Coulomb pseudopotential (for simple metals $\mu^{*} \cong 0.13$ ).

Let us consider an amorphous binary alloy containing simple metals. Then, the electron-ion interaction is described within the pseudopotential concept [27]. Topological disorder is present in an amorphous alloy which means that ion quasiequilibrium positions are irregular and ions vibrate near these positions. The Eliashberg function has the following form within the second order of the perturbation theory in pseudopotential $[20-22,26]$

$$
\begin{aligned}
& \alpha^{2} g(\omega)=-\frac{1}{\pi \hbar}\left[\int_{S_{\mathrm{F}}} \frac{\mathrm{d}^{2} k}{\hbar V_{k}}\right]^{-1} \int_{S_{\mathrm{F}}} \frac{\mathrm{d}^{2} k}{\hbar V_{k}} \int_{S_{\mathrm{F}}} \frac{\mathrm{d}^{2} k^{\prime}}{\hbar V_{k}} \\
& \quad \times \sum_{\alpha \beta} \frac{C_{\alpha} W_{\alpha}\left(k-k^{\prime}\right) C_{\beta} W_{\beta}\left(k-k^{\prime}\right)}{\Omega_{0}} \operatorname{Im} G_{\alpha \beta}\left(k-k^{\prime}, \omega\right) .
\end{aligned}
$$

Here $W_{\alpha}(k)$ is a form factor of screened electron-ion pseudopotential, subscript $\alpha(\alpha=1,2)$ indicates a kind of atoms; $N_{\alpha}, C_{\alpha}=N_{\alpha} / N$ are a number of $\alpha$-kind atoms and their concentration, respectively, $N=N_{1}+N_{2}$ - a total number of atoms in an alloy, $\Omega_{0}=\Omega / N$ - volume per one atom and $V_{\boldsymbol{k}}$ is an electron velocity. Integration in (3) is performed over the Fermi-surface $S_{\mathrm{F}}$. The function $G_{\alpha \beta}(q, \omega)$ entering Eq. (3) is the Fourier-transform of the retarded phonon Green function

$$
G_{\alpha \beta}(q, t)=\mathrm{i} \hbar \Theta(t) \overline{\left\langle\left[\rho_{\alpha}(\boldsymbol{q}, t): \rho_{\beta}^{+}(q, 0)\right]\right\rangle}
$$

with $\Theta(t)$ the step-function and

$$
\rho_{\alpha}(q, t)=\frac{1}{N_{\alpha}} \sum_{\nu_{\alpha}=1}^{N_{\alpha}}\left(\mathrm{i} q \boldsymbol{\xi}_{\nu_{\alpha}}(t)\right) \exp \left(\mathrm{i} q \boldsymbol{R}_{0 \nu_{\alpha}}(t)\right),
$$

where $\xi_{\nu_{\alpha}}(t)$ is a displacement of the $\nu_{\alpha}$-ion in time $t$ from the equilibrium position $\boldsymbol{R}_{0 \nu_{\alpha}}(t)$. Angular brackets in (4) stand for the Gibbs averaging over the vibrational degrees of freedom while the line over the expression means the configurational averaging. One must know the atomic distribution functions to perform the configurational averaging over the quasiequilibrium ion positions in (4). A model of a. frozen liquid $[20,21,28,29]$ is exploited to describe a structure of amorphous 
system in the present paper. According to this model the structure of the amorphous state is determined within the formalism of the correlation functions. The last are defined likewise in the theory of liquids [30, 31]. For example, the binary partial correlation functions are determined by equations $[30,31]$

$$
g_{\alpha \beta}\left(\boldsymbol{r}_{1}-\boldsymbol{r}_{2}\right)=\frac{\Omega^{2}}{N_{\alpha} N_{\beta}} \overline{\sum_{j_{\alpha}}^{N_{\alpha}} \sum_{j_{\beta}=1}^{N_{\beta}} \delta\left(\boldsymbol{R}_{j_{\alpha}}-r_{1}\right) \delta\left(\boldsymbol{R}_{j_{\beta}}-\boldsymbol{r}_{1}\right)},
$$

where $j_{\alpha} \neq j_{\beta}, \alpha, \beta=1,2$. The $g_{\alpha \beta}\left(r_{1}-r_{2}\right)$ functions are parameters of the amorphous state theory [28]. A model of hard spheres is generally utilized in numerical illustrations of this theory $[28,30,31]$. To perform calculations we also use the partial correlation functions of a binary system of hard spheres [31] as $g_{\alpha \beta}\left(r_{1}-r_{2}\right)$.

A detailed investigation of the phonon Green function for amorphous pure metal was carried out in Ref. [28], where the dispersion equation was obtained and it was shown that for $q a<1$, the attenuation of the phonon spectrum was weak and tended to zero as $(q a)^{4}$, where $a$ is a mean distance between atoms.

Generalization of this technique to the case of amorphous alloy does not face any difficulties. Then, the phonon Green function $G_{\alpha \beta}(q, t)$ takes the following form if one neglects the attenuation mentioned above:

$$
\begin{aligned}
& G_{\alpha \beta}(q, t)=\mathrm{i} \hbar \int \mathrm{d} \omega \exp (\mathrm{i} t \omega) \sum_{k} \sum_{\gamma} \frac{\left[q \varepsilon_{\alpha}(k, \gamma)\right]\left[q \varepsilon_{\beta}(k, \gamma)\right]}{2 N \bar{M} \omega(k, \gamma)} \\
& \quad \times\left[N \Delta(k+q)+S_{\alpha \beta}(k+q)\right]\left[\frac{1}{\omega-\omega(k, \gamma)-\mathrm{i} \delta}-\frac{1}{\omega+\omega(k, \gamma)-\mathrm{i} \delta}\right] .
\end{aligned}
$$

Here $\bar{M}=C_{1} M_{1}+C_{2} M_{2}$ is the average ion mass, the vector $\varepsilon_{\alpha}(k, \gamma)$ and frequency $\omega(k, \gamma)$ determine the polarization states of vibrational excitations and their spectrum, respectively, $\gamma=1,2, \ldots, 6$ polarization and $k$ - the wave vector. At last, $S_{\alpha \beta}(q), \alpha, \beta=1,2$ are the partial structure factors of amorphous binary alloy defined as follows:

$$
\frac{N}{N_{\alpha} N_{\beta}} \overline{\sum_{j_{\alpha}=1}^{N_{\alpha}} \sum_{j_{\beta}=1}^{N_{\beta}} \exp \left[\mathrm{i} \boldsymbol{q}\left(\boldsymbol{R}_{0 j_{\alpha}}-\boldsymbol{R}_{0 j_{\beta}}\right)\right]}=N \Delta(q)+S_{\alpha \beta}(q),
$$

where

$$
\Delta(k)=\left\{\begin{array}{lll}
1 & \text { for } & k=0 \\
0 & \text { for } & k \neq 0
\end{array}\right.
$$

The line over expression in the left-hand side of Eq. (8) means configurational averaging. The partial structure factors $S_{\alpha \beta}(q)$ are connected with the partial binary correlation functions by equations

$$
S_{\alpha \beta}(q)-\delta_{\alpha \beta}=\frac{1}{\Omega_{0}} \int \mathrm{d}^{3} r \exp (-\mathrm{i} \boldsymbol{q} r) g_{\alpha \beta}(r) .
$$

Here $\delta_{\alpha \beta}$ is the Kronecker symbol.

The Fourier transform of the phonon Green function $G_{\alpha \beta}(q, \omega)$ is substituted in Eq. (3) after transition to the thermodynamic limit $N, \Omega \rightarrow \infty, \Omega_{0}=\Omega / N=$ const. 
The electron-phonon coupling constant $\lambda$ takes the final form after utilizing Eqs. (1) and (3)

$$
\lambda=\lambda_{0}+\lambda_{1}
$$

with

$$
\begin{aligned}
\lambda_{0}= & \left(\int_{S_{\mathrm{F}}} \frac{\mathrm{d}^{2} k}{\hbar V_{k}}\right)^{-1} \int_{S_{\mathrm{F}}} \frac{\mathrm{d}^{2} k}{\hbar V_{k}} \int_{S_{\mathrm{F}}} \frac{\mathrm{d}^{2} k^{\prime}}{\hbar V_{k^{\prime}}} \sum_{\alpha, \beta} \frac{C_{\alpha} W_{\alpha}\left(k-k^{\prime}\right) C_{\beta} W_{\beta}\left(k-k^{\prime}\right)}{\Omega_{0}} \\
& \times \sum_{\gamma} \frac{\left[\left(k-k^{\prime}\right) \varepsilon_{\alpha}\left(k-k^{\prime}, \gamma\right)\right]\left[\left(k-k^{\prime}\right) \varepsilon_{\beta}\left(k-k^{\prime}, \gamma\right)\right]}{\bar{M} \omega^{2}\left(k-k^{\prime}, \gamma\right)}
\end{aligned}
$$

and

$$
\begin{aligned}
& \lambda_{1}=\left(\int_{S_{\mathrm{F}}} \frac{\mathrm{d}^{2} k}{\hbar V_{k}}\right)^{-1} \int_{S_{\mathrm{F}}^{\prime}} \frac{\mathrm{d}^{2} k}{\hbar V_{k}^{\prime}} \int_{S_{\mathrm{F}}} \frac{\mathrm{d}^{2} k^{\prime}}{\hbar V_{k^{\prime}}} \sum_{\alpha, \beta} \frac{C_{\alpha} W_{\alpha}\left(k-k^{\prime}\right) C_{\beta} W_{\beta}\left(k-k^{\prime}\right)}{\Omega_{0}} \\
& \times \int \frac{\mathrm{d}^{3} k_{1}}{(2 \pi)^{3}} S_{\alpha \beta}\left(k_{1}+k-k^{\prime}\right) \sum_{\gamma} \frac{\left[\left(k-k^{\prime}\right) \varepsilon_{\alpha}\left(k_{1}, \gamma\right)\right]\left[\left(k-k^{\prime}\right) \varepsilon_{\beta}\left(k_{1}, \gamma\right)\right]}{\bar{M} \omega^{2}\left(k_{1}, \gamma\right)} .
\end{aligned}
$$

Functions $\omega(k, \gamma)$ and $\varepsilon(k, \gamma)$ entering in Eqs. (11) and (12) obey the following system of equations, which can be obtained after diagonalization of the phonon part of the system Hamiltonian $[21,29]$ :

$$
\sum_{\beta} \sum_{m} \sqrt{C_{\beta} M_{\beta}} F_{\alpha \beta}^{l m}(k) \varepsilon_{\beta}^{m}(k, \gamma)=\omega^{2}(k, \gamma) \sqrt{C_{\alpha} M_{\alpha}} \varepsilon_{\alpha}^{l}(k, \gamma) .
$$

Here $l, m=x, y, z$ are Cartesian coordinates, $M_{\alpha}$ - the mass of an $\alpha$-kind ion and

$$
\begin{aligned}
& F_{\alpha \beta}^{l m}(k)=\frac{\Omega_{0}}{(2 \pi)^{3} \sqrt{C_{\alpha} M_{\alpha} C_{\beta} M_{\beta}}} \int \mathrm{d}^{3} k_{1} k_{1}^{l} k_{1}^{m}\left[\Phi_{\alpha \beta}\left(k_{1}\right) C_{\alpha} C_{\beta} S_{\alpha \beta}\left(k+k_{1}\right)\right. \\
& \left.\quad-\delta_{\alpha \beta} \sum_{\sigma} \Phi_{\alpha \sigma}\left(k_{1}\right) C_{\alpha} C_{\sigma} S_{\alpha \sigma}\left(k_{1}\right)\right]
\end{aligned}
$$

are the elements of the dynamic matrix with $\Phi_{\alpha \beta}(k)$ the Fourier transform of ion-ion effective interaction.

One can find the spectrum of phonon excitations solving the eigenvalues problem (13). There are the acoustic and optical branches in the phonon excitation spectrum of amorphous alloy $[21,29,32]$.

The tuansverse waves have the form

$$
\begin{gathered}
\omega_{\mathrm{ta}}^{2}(k)=\omega^{2}(k, 1)=\omega^{2}(k, 2)=\frac{1}{2}\left[F_{11}(k)+F_{22}(k)\right] \\
-\sqrt{\frac{1}{2}\left[F_{11}^{x x}(k)-F_{22}^{x x}(k)\right]^{2}+\left[F_{12}^{x x}(k)\right]^{2}}
\end{gathered}
$$

for the acoustic modes and 


$$
\begin{gathered}
\omega_{\text {to }}^{2}(k)=\omega^{2}(k, 3)=\omega^{2}(k, 4)=\frac{1}{2}\left[F_{11}^{x x}(k)+F_{22}^{x x}(k)\right] \\
-\sqrt{\frac{1}{4}\left[F_{11}^{x x}(k)-F_{22}^{x x}(k)\right]^{2}+\left[F_{12}^{x x}(k)\right]^{2}}
\end{gathered}
$$

for the optical ones.

The longitudinal waves have the following form:

$$
\begin{aligned}
& \omega_{\mathrm{la}}^{2}(k)=\omega^{2}(k, 5)=\frac{1}{2}\left[F_{11}^{z z}(k)+F_{22}^{z z}(k)\right] \\
&-\sqrt{\frac{1}{4}\left[F_{11}^{z z}(k)-F_{22}^{z z}(k)\right]^{2}+\left[F_{12}^{\hat{z}}(k)\right]^{2}}
\end{aligned}
$$

for the acoustic mode and

$$
\begin{gathered}
\omega_{\mathrm{lo}}^{2}(k)=\omega^{2}(k, 6)=\frac{1}{2}\left[F_{11}^{z z}(k)+F_{22}^{z z}(k)\right] \\
-\sqrt{\frac{1}{4}\left[F_{11}^{z z}(k)-F_{22}^{z z}(k)\right]^{2}+\left[F_{12}^{z z}(k)\right]^{2}}
\end{gathered}
$$

for the optical one.

The frequencies $\omega(k, \gamma)$ and the corresponding vectors of polarization $\varepsilon_{\alpha}^{m}(k, \gamma)$ are the known quantities after solving the eigenvalues problem (13). Then, it is easy to calculate the following sums:

$$
\sum_{\gamma} \frac{\left[q \varepsilon_{\alpha}(k, \gamma)\right]\left[q \varepsilon_{\beta}(k, \gamma)\right]}{\omega^{2}(k, \gamma)}=\left(q_{x}^{2}+q_{y}^{2}\right) D_{\alpha \beta}^{x x}(k)+q_{z}^{2} D_{\alpha \beta}^{z z}(k),
$$

where

$$
\begin{aligned}
& D_{11}^{z z}=\frac{F_{22}^{z z}-C_{2} \omega_{\mathrm{la}}^{2}}{C_{1}\left[F_{11}^{z z} F_{22}^{z z}-\left(F_{12}^{z z}\right)^{2}\right]} \\
& D_{22}^{z z}=\frac{F_{11}^{z z}-C_{1} \omega_{\mathrm{la}}^{2}}{C_{2}\left[F_{11}^{z z} F_{22}^{z z}-\left(F_{12}^{z z}\right)^{2}\right]}, \\
& D_{12}^{z z}=D_{21}^{z z}=\frac{-F_{22}^{z z}+\sqrt{C_{1} C_{2}} \omega_{\mathrm{la}}^{2}}{\sqrt{C_{1} C_{2}}\left[F_{11}^{z z} F_{22}^{z z}-\left(F_{12}^{z z}\right)^{2}\right]}
\end{aligned}
$$

and

$$
\begin{aligned}
& D_{11}^{x x}=D_{11}^{y y}=\frac{F_{22}^{x x}-C_{2} \omega_{\mathrm{ta}}^{2}}{C_{1}\left[F_{11}^{z z} F_{22}^{x x}-\left(F_{12}^{x x}\right)^{2}\right]} \\
& D_{22}^{x x}=D_{22}^{y y}=\frac{F_{11}^{x x}-C_{1} \omega_{\mathrm{ta}}^{2}}{C_{2}\left[F_{11}^{z z} F_{22}^{x x}-\left(F_{12}^{x x}\right)^{2}\right]} \\
& D_{12}^{x x}=D_{21}^{x x}=D_{12}^{y y}=D_{21}^{y y}=\frac{-F_{12}^{m k}+\sqrt{C_{1} C_{2}} \omega_{\mathrm{ta}}^{2}}{\sqrt{C_{1} C_{2}}\left[F_{11}^{z z} F_{22}^{x x}-\left(F_{12}^{x x}\right)^{2}\right]} \\
& D_{\alpha \beta}^{l m}=0 \quad \text { when } \quad l \neq m .
\end{aligned}
$$


Substituting the obtained expressions (19)-(22) into (11) and (12) and integrating over the angles we get the final formulae for $\lambda_{0}$ and $\lambda_{1}$ convenient for numerical calculations

$$
\begin{aligned}
& \lambda_{0}=12 \frac{m Z_{0}}{\bar{M}} \int_{0}^{1} \frac{z^{3} \mathrm{~d} z}{h^{2} \Omega_{0}^{2}} \sum_{\alpha, \beta} C_{\alpha} W_{\alpha}\left(2 k_{\mathrm{F}} z\right) D_{\alpha \beta}^{z z}\left(2 k_{\mathrm{F}} z\right) C_{\beta} W_{\beta}\left(2 k_{\mathrm{F}} z\right) \\
& \lambda_{1}=72 \frac{m Z_{0}}{\bar{M}} \int_{0}^{1} \frac{z^{3} \mathrm{~d} z}{h^{2} \Omega_{0}^{2}} \int_{0}^{\left(4 Z_{0}\right)^{-1 / 3}} y^{2} \mathrm{~d} y \int_{-1}^{1} \mathrm{~d} x \sum_{\alpha, \beta} C_{\alpha} W_{\alpha}\left(2 k_{\mathrm{F}} z\right) C_{\beta} W_{\beta}\left(2 k_{\mathrm{F}} z\right) \\
& \times S_{\alpha \beta}\left(2 k_{\mathrm{F}} \sqrt{z^{2}+y^{2}+2 x y z}\right)\left[x^{2} D_{\alpha \beta}^{z z}\left(2 k_{\mathrm{F}} y\right)+\left(1-x^{2}\right) D_{\alpha \beta}^{z z}\left(2 k_{\mathrm{F}} y\right)\right]
\end{aligned}
$$

Here $m$ is the electron mass, $Z_{0}=C_{1} z_{1}+C_{2} z_{2}$ - the average valency with $z_{\alpha}$ $(\alpha=1,2)$ the valency of pure metals ions and $k_{\mathrm{F}}$ is the Fermi vector.

One should emphasize that in the limit $C \rightarrow 0$ or $C \rightarrow 1$ the obtained formulae (23) and (24) take the form of appropriate equations for $\lambda_{0}$ and $\lambda_{1}$ derived in Refs. [20-22] for the case of a pure amorphous metal.

\section{Numerical calculations and discussion}

The structure factor of an ideal crystal is defined by the following equation:

$$
S(k)=\frac{(2 \pi)^{3}}{\Omega_{0}} \sum_{k_{n}} \delta\left(k-k_{n}\right)
$$

where $k_{n}$ is reciprocal lattice vectors. Substituting Eq. (25) into (10) it is easy to prove that the term $\lambda_{1}(24)$ is equal to zero in an ideal crystal case and $\lambda_{0}(23)$ has a standard form like in Ref. [26]. In amorphous alloys the structure factor $S(k)$ is substantially different from (25) because of the topological disorder. Therefore, the $\lambda_{1}$ term does not equal zero and the constant of the electron-phonon coupling $\lambda(10)$ is larger in an amorphous alloy than in a crystalline one. Besides, it follows from the analysis of Eqs. (10)-(12), that the $\lambda$ constant of amorphous alloy can be larger than the $\lambda$ of pure amorphous metals which form this alloy because of additional contributions arising from interactions between electrons and optical phonons. Direct numerical calculations confirm this statement. Let us detail them.

The structure factor of a binary system of hard spheres, obtained within the Perkus-Yewick approximation $[30,31]$ is used in calculations. The effective ion-ion potentials and the electron-phonon interactions are considered with utilizing the Ashcroft model pseudopotential [33]. It has been shown in Ref. [22] for a pure amorphous metal case that the calculated value of the superconducting transition temperature $T_{c}$ depends essentially on the Ashcroft pseudopotential parameter $R_{\mathrm{c}}$. Therefore, in order to investigate $T_{\mathrm{c}}$ dependence on alloy concentration the following method is used for determining parameters $R_{\mathrm{c} \alpha}(\alpha=1,2)$ of the alloy components Ashcroft pseudopotential. The $R_{\mathrm{c} \alpha}(\alpha=1,2)$ parameter is found from the condition that the calculated and experimental values of $T_{\mathrm{c}}$ must coincide for a pure amorphous metal. Then $T_{c}$ as a function of alloy concentration is evaluated. The results of calculations are presented for some amorphous binary alloys in Figs. 1-6. Figures 1 and 2 are especially interested, because some experimental data on $T_{\mathrm{c}}=f(c)$ are available for $\mathrm{BeCu}$ and $\mathrm{AlCu}$ amorphous alloys [34, 35]. 

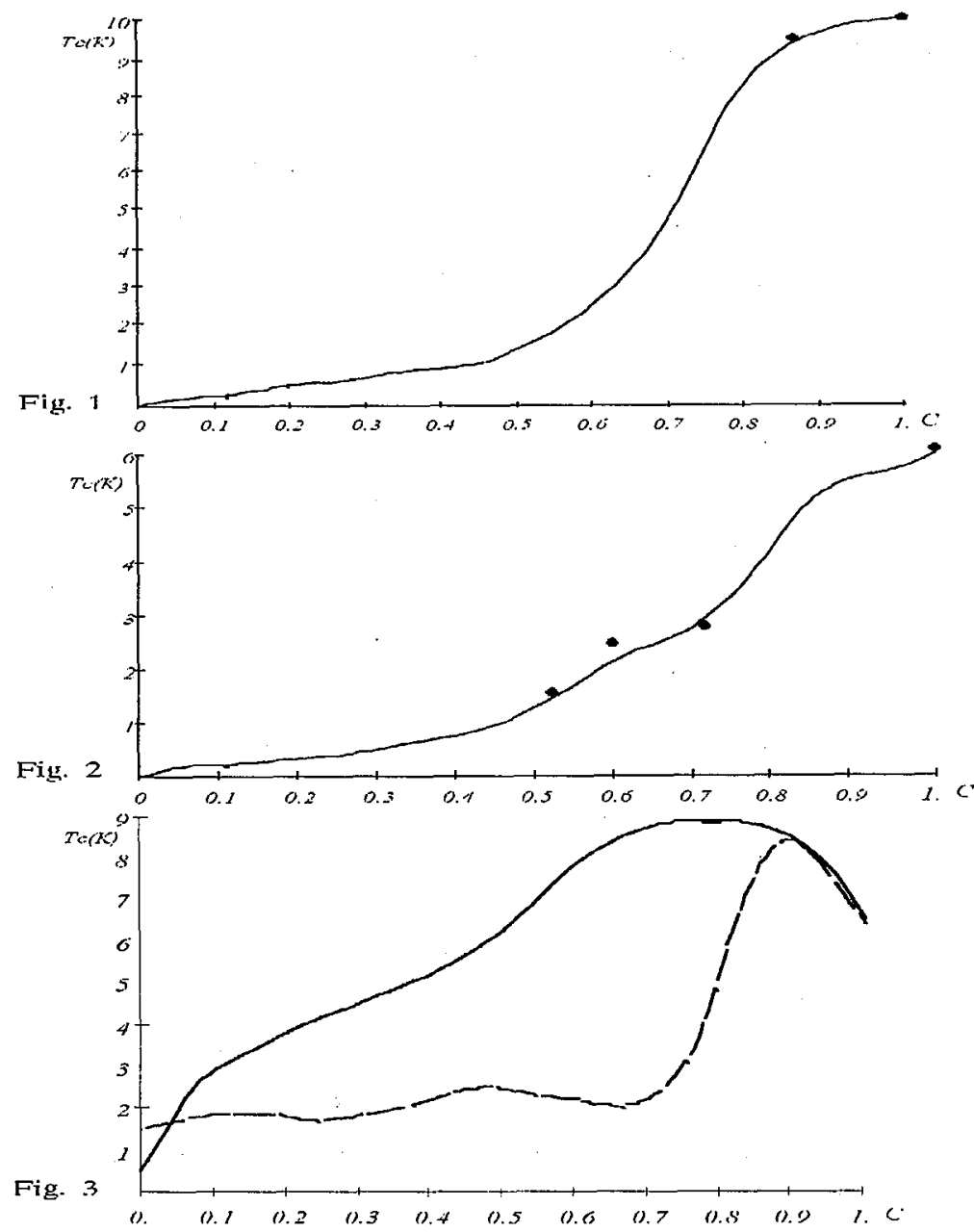

Fig. 1. Dependence of $T_{\mathrm{c}}$ on the concentration $C$ of the first metal for BeCu alloy.

Fig. 2. The same as in Fig. 1 for AlCu amorphous alloy.

Fig. 3. Dependence of $T_{c}$ on the concentration $C$ of the first metal in AlZn (dashed line) and AlMg (solid line) alloys.

These data are indicated with the rhombus-symbols in the figures. It is seen that agreement between theory and experiment is very good. This fact allows us to extend the developed approach to amorphous binary systems for which the experimental data on $T_{\mathrm{c}}$ dependence on concentration are absent. The results of such numerical investigations are shown in Figs. 3 to 6 . It is seen from Figs. 3 to 6 that $T_{\mathrm{c}}$ as a function of alloy concentration has a maximum in almost all investigated systems. There is an interval of concentration in AlMg, AlZn, LiAl, LiGa, and $\mathrm{MgZn}$ alloys in which the values of the superconducting transition temperature exceed $T_{c}$ in pure metals forming these alloys, see Figs. 3-6. 


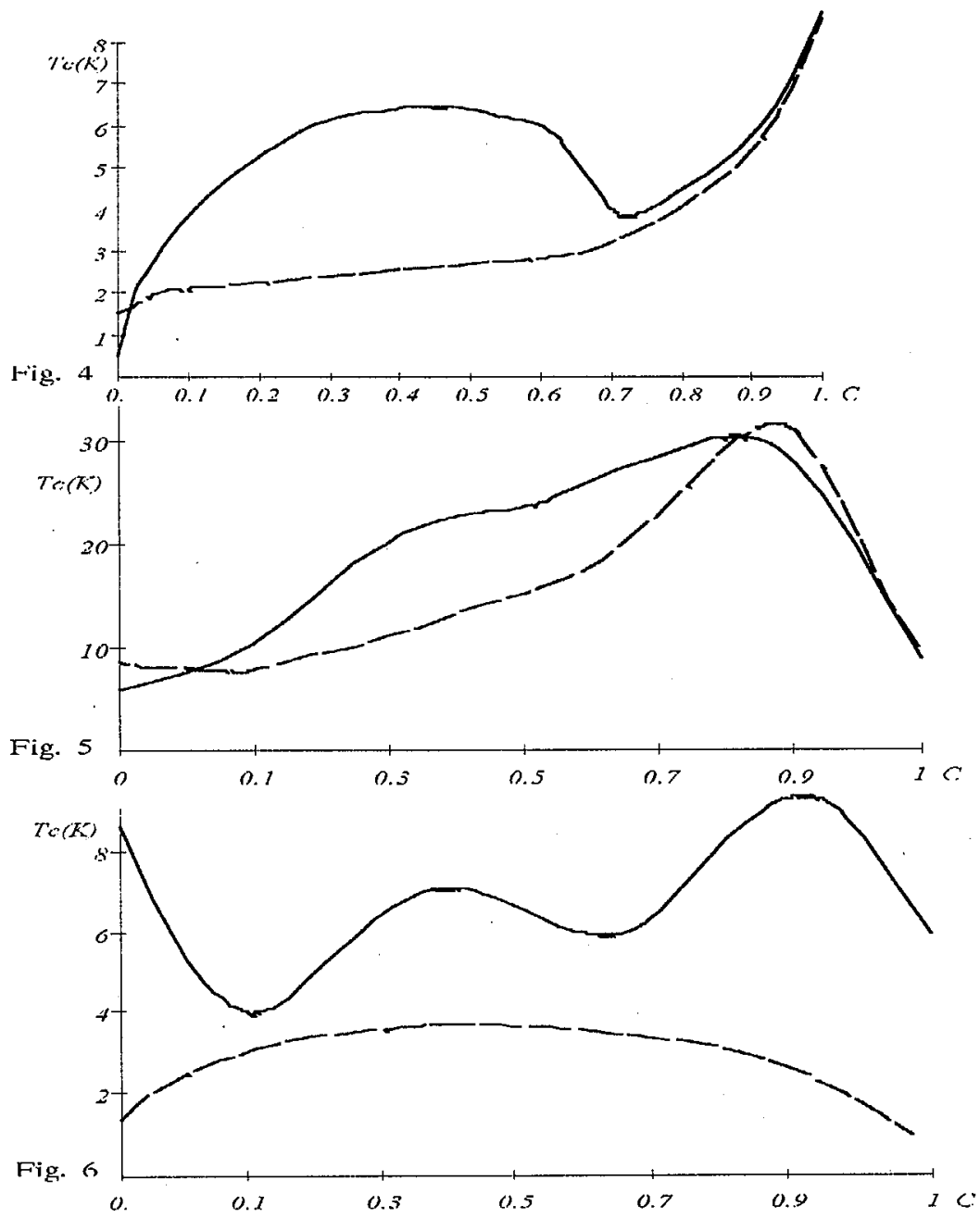

Fig. 4. The same as in Fig. 3 for GaZn (dashed line) and GaMg (solid line) alloys. Fig. 5. The same as in Fig. 1 for $\mathrm{LiGa}$ (dashed line) and LiAl (solid line) alloys. Fig. 6. The same as in Fig. 1 for $\mathrm{MgZn}$ (dashed line) and $\mathrm{AlGa}$ (solid line) alloys.

\section{Acknowledgments}

The authors would like to thank the referee for careful reading of the manuscript and suggested corrections. We would like to acknowledge the fruitful discussions with Prof. Z. Gurski.

\section{References}

[1] W. Buckel, R. Hilsch, Z. Phys. 131, 420 (1952); ibid. 138, 109 (1954).

[2] G. von Minnigerode, Z. Phys. 154, 442 (1959).

[3] E. Strieder, Ann. Phys. 22, 15 (1968).

[4] G. von Minnigerode, J. Rothenberg, Z. Phys. 213, 397 (1968). 
[5] K. Knorr, N. Barth, Solid State Commun. 6, 791 (1968).

[6] G. Bergmann, Z. Phys. 228, 25 (1969).

[7] G. Ziemba, G. Bergmann, Z. Phys. 237, 410 (1970).

[8] A. Comberg, S. Ewert, Z. Phys. 268, 241 (1974).

[9] A. Comberg, S. Ewert, G. Bergmann, Z. Phys. 271, 317 (1974).

[10] G. Bergmann, Phys. Rep. 27, 159 (1976).

[11] V.V. Nemoshkalenko, A.V. Romanova, A.G. Il'insky, V.V. Maslov, D.Yu. Paderno, Yu.V. Korniushyn, V.G. Nikityn, M.E. Osinowski, I.Ya. Diechtiar, E.G. Madotova, M.A. Vasiliev, D.J. Bratus, V.M. Pan, A.E. Shpigiel, M.P. Voronko, in: Amorphous Metalic Alloys, Ed. V.V. Nemoshkalenko, Naukova Dumka, Kiev 1987 (in Russian).

[12] M. Gurvitch, A.K. Ghosh, B.L. Gyorffy, H. Lutz, O.F. Kammerer, J.S. Rosner, M. Strongin, Phys. Rev. Lett. 41, 1616 (1978).

[13] R.C. Dynes, I.P. Garno, Phys. Rev. Lett. 46, 137 (1981).

[14] B. Keck, A. Schmid, J. Low. Temp. Phys. 24, 611 (1976).

[15] P.W. Anderson, K.A. Muttalib, T.V. Ramakrishnan, Phys. Rev. B 28, 117 (1983).

[16] H. Fukuyama, H. Ebisawa, S. Maekawa, J. Phys. Soc. Jpn. 53, 1919 (1984); ibid. 53, 3560 (1984).

[17] D. Belitz, J. Phys. F 15, 2315 (1985).

[18] D. Belitz, Phys. Rev. B 35, 1637 (1987); ibid. 35, 1651 (1987).

[19] M.V. Sadovskii, Phys. Rep. 282, 225 (1997).

[20] Yu.P. Krasny, N.P. Kovalenko, Physica B 162, 128 (1990).

[21] Yu.P. Krasny, N.P. Kovalenko, V.V. Micho, J. Mol. Liquids 58, 45 (1993).

[22] Yu.P. Krasny, N.P. Kovalenko, V.A. Tesis, Mol. Phys. Rep. 11, 143 (1995).

[23] J.M. Ziman, Philos. Mag. 6, 1013 (1961).

[24] E.G. Maximow, Zh. Exsp. Teor. Fiz. (JETP) 57, 1660 (1969) (in Russian).

[25] W.J. McMillan, Phys. Rev. 167, 331 (1968).

[26] S.V. Vonsovski, Yu.A. Izumov, Superconductivity of Transition Metals, their Alloys and Compounds, Nauka, Moscow 1977 (in Russian).

[27] W.A. Harrison, Pseudopotentials in the Theory of Metals, W.A. Benjamin Inc., New York 1965.

[28] I.A. Vakarchuk, V.M. Myhal, V.M. Tkachuk, Teor, Mat. Fiz. 75, 306 (1988) (in Russian).

[29] N.P. Kovalenko, Yu.P. Krasny, Physica B 162, 115 (1990).

[30] D.K. Belastshenko, Structure of Liquid and Amorphous Metals, Metallurgiya, Moscow 1985 (in Russian).

[31] E.I. Khar'kov, V.I. Lysow, M.A. Fyodorov, Physics of Liquid Metals, Vystsha Shkola, Kiev 1985 (in Russian).

[32] N.P. Kovalenko, Yu.P. Krasny, G. Weck, Phys. Status Solidi B 166, 117 (1991),

[33] N.W. Ashcroft, D.C. Langreth, Phys. Rev. 155, 682 (1967); ibid. 159, 500 (1967).

[34] W.H.-G. Müller, Z. Phys. B 40, 203 (1981).

[35] A. Lambrecht, H. Leitz, J. Hasse, Z. Phys. B 40, 35 (1981). 